\title{
Experimental infection of cats with Tritrichomonas foetus
}

\author{
Jody L. Gookin, DVM, PhD; Michael G. Levy, PhD; J. Mac Law, DVM, PhD; \\ Mark G. Papich, DVM, MS; Matthew F. Poore; Edward B. Breitschwerdt, DVM
}

\begin{abstract}
Objective-To determine whether infection with Tritrichomonas foetus causes diarrhea in specificpathogen-free or Cryptosporidium coinfected cats.
\end{abstract}

Animals-4 cats with subclinical cryptosporidiosis (group 1) and 4 specific-pathogen-free cats (group 2).

Procedure-Cats were infected orogastrically with an axenic culture of $T$ foetus isolated from a kitten with diarrhea. Direct microscopy and protozoal culture of feces, fecal character, serial colonic mucosal biopsy specimens, and response to treatment with nitazoxanide (NTZ; group 1) or prednisolone (groups 1 and 2) were assessed.

Results - Infection with $T$ foetus persisted in all cats for the entire 203-day study and resulted in diarrhea that resolved after 7 weeks. Group-1 cats had an earlier onset, more severe diarrhea, and increased number of trichomonads on direct fecal examination, compared with group-2 cats. Use of NTZ eliminated shedding of $T$ foetus and Cryptosporidium oocysts, but diarrhea consisting of trichomonad-containing feces recurred when treatment was discontinued. Prednisolone did not have an effect on infection with $T$ foetus but resulted in reappearance of Cryptosporidium oocysts in the feces of 2 of 4 cats. During necropsy, $T$ foetus was isolated from contents of the ileum, cecum, and colon. Tritrichomonas foetus organisms and antigen were detected on surface epithelia and within superficial detritus of the cecal and colonic mucosa.

Conclusions and Clinical Relevance-After experimental inoculation in cats, $T$ foetus organisms colonize the ileum, cecum, and colon, reside in close contact with the epithelium, and are associated with transient diarrhea that is exacerbated by coexisting cryptosporidiosis but not treatment with prednisolone. (Am J Vet Res 2001;62:1690-1697)

$\mathrm{T}$ richomonads have been infrequently reported in association with diarrhea in cats. In the early 1900s, Pentatrichomonas spp were described as naturally occurring organisms in the feces of cats. ${ }^{1-5}$ In those studies, clinical signs of infection were not evident or were difficult to ascribe solely to trichomonosis. Current views of intestinal trichomonosis in cats are

Received Jan 22, 2001.

Accepted Mar 28, 2001.

From the Departments of Anatomy, Physiological Sciences and Radiology (Gookin, Papich), Microbiology, Pathology and Parasitology (Levy, Law, Poore), and Clinical Sciences (Breitschwerdt), College of Veterinary Medicine, North Carolina State University, Raleigh, NC 27606.

Presented in part at the American College of Veterinary Internal Medicine Forum, May 2000, Seattle, Wash

The authors thank Julie Bradley, Beth Salmon, and Drs. Jim Guy and Karine Savary for technical assistance. strongly biased by those early studies, suggesting that trichomonads are nonpathogenic commensal fauna that flourish only when there is coexisting enteric disease. ${ }^{6-9}$ Perhaps because of this presumption, there is only 1 report $^{10}$ of a cat with intestinal trichomonosis and diarrhea during the 60-year period prior to 1996. During the past 4 years, an increased incidence of young cats with large-bowel diarrhea in which the feces contained large numbers of trichomonads has been reported. ${ }^{11-13}$ Infected cats often were from catteries or shelters, were frequently misdiagnosed as infected with Giardia organisms, had a waxing and waning course of diarrhea that failed to respond to antigiardial treatment, and were apparently free of concurrent intestinal disease or infection. ${ }^{11}$ Because attempts to culture trichomonads from the feces of healthy cats have failed, ${ }^{11}$ these organisms do not appear to be a component of the normal intestinal fauna. Although initially presumed to be Pentatrichomonas hominis, ${ }^{11-13}$ we recently cloned the 18S rRNA gene of several naturally occurring isolates obtained from cats that, surprisingly, has $99.9 \%$ sequence identity to the DNA of Tritrichomonas foetus, ${ }^{14,15}$ the organism that is responsible for venereal trichomonosis in cattle and is found in the intestinal tract of wild and domestic swine. ${ }^{16,17}$ Consistent with the failures of antimicrobial treatment of $T$ foetus in cattle with venereal infection, cats with diarrhea and trichomonosis have been refractory to antimicrobial therapy. ${ }^{11-13}$ An in vitro susceptibility assay of the T foetus isolate (NCSU Tfs-1) used in the study reported here revealed resistance to all tested antimicrobials, except for nitazoxanide (NTZ), ${ }^{a}$ a drug with broad-spectrum antimicrobial activity. ${ }^{18}$

We hypothesized that $T$ foetus may represent a newly recognized intestinal pathogen that causes largebowel diarrhea in naturally infected cats. Therefore, the objectives of the study reported here were to determine whether a naturally occurring isolate of feline $T$ foetus could cause diarrhea in specific-pathogen-free (SPF) cats and to determine whether the clinical manifestations of infection attributable to $T$ foetus were influenced by coexisting cryptosporidial infection or immunosuppression. We also sought to examine the in vivo efficacy of NTZ, a drug with documented in vitro activity against the $T$ foetus isolate used in the study. ${ }^{a}$

\section{Materials and Methods}

Cats-Eight 4-month-old sexually intact female domestic shorthair cats were purchased from a commercial vendor. ${ }^{b}$ Four cats were from a conventionally reared and vaccinated colony (group 1), and 4 cats were from a SPF and unvaccinated colony (group 2). At the time of initial evaluation, small numbers of Cryptosporidium oocysts were identified in the feces of all cats in group 1. 
Each group was housed in a separate room, and all cats within a room were in separate cages and received the same dry food ${ }^{c}$ ad libitum throughout the study. Cages were cleaned daily and disinfected with a $2 \%$ solution of chlorhexidine. Cats were housed under conditions of controlled lighting and temperature and were maintained in compliance with biosafety level-2 guidelines. The protocol was approved by the North Carolina State University Institutional Animal Care and Use Committee.

During an initial 2-week acclimatization period, each cat was determined to be healthy on the basis of results of physical examination, CBC, serum biochemical analyses, urinalysis, measurement of pre- and postprandial serum bile acid concentrations, and tests for FeLV, feline immunodeficiency virus (FIV), and feline enteric coronavirus. Other than cryptosporidiosis in group-1 cats, other enteric pathogens were not identified by repeated analyses of fecal samples, including a flotation method for parasite ova, direct microscopy for protozoa ( $\mathrm{n}=3$ examinations/cat) and Campylobacter spp, Gram stain for Clostridium spp, microbial culture for Tritrichomonas spp (3 examinations/cat) and Salmonella spp, antigen test for Giardia spp, ${ }^{\mathrm{d}}$ and transmission electron microscopy for viruses. Fluorescence microscopy of fecal smears stained with Auramine-0 (3 examinations/cat) revealed a small number of Cryptosporidium oocysts in feces from all group-1 cats. Cryptosporidium oocysts were not seen in the feces of any group-2 cats. Results of histologic examination of colonic mucosal biopsy specimens obtained from the proximal, middle, and distal portions of the colon from all cats prior to inoculation were considered normal.

Isolation of $T$ foetus - Trichomonads were isolated from a fecal sample obtained from a 6-month-old sexually intact female domestic longhair cat. The cat had a history of chronic large-bowel diarrhea that was first detected when the cat was 6 weeks old. Despite treatment with recommended dosages of pyrantel pamoate, sulfadimethoxine, and metronidazole, the feces contained an unusually large number of motile trichomonads. The cat was otherwise healthy, as determined on the basis of results of physical examination, $\mathrm{CBC}$, serum biochemical analyses, urinalysis, measurement of pre- and postprandial serum bile acid concentrations, and tests for FeLV, FIV, and feline enteric coronavirus. Other enteric pathogens were not detected by analyses of repeated fecal samples, including a flotation method for parasite ova, direct microscopy for nontrichomonad protozoa and Campylobacter spp, Gram stain for Clostridium spp, microbial culture for Salmonella spp, antigen test for Giardia spp, ${ }^{\mathrm{d}}$ Auramine-0 stain and fluorescence microscopy for Cryptosporidium oocysts, and transmission electron microscopy for viruses. Moderate to severe diffuse lymphocytic-plasmacytic colitis was observed during histologic examination of sections of colonic mucosal biopsy specimens obtained from the proximal, middle, and distal portions of the colon.

Approximately $0.1 \mathrm{ml}$ of freshly voided feces was suspended in $10 \mathrm{ml}$ of PBS solution ( $\mathrm{pH} \mathrm{7.3).} \mathrm{A} \mathrm{100- \mu l} \mathrm{aliquot}$ of the suspension then was inoculated into a culture tube containing $10 \mathrm{ml}$ of antibiotic-fortified $\left(10^{6}\right.$ units of penicillin/L, $15 \mathrm{~g}$ of streptomycin/L, $2 \mathrm{mg}$ of amphotericin B/L) modified Diamond medium ${ }^{e}$ and incubated at 37 C. A $100-\mu l$ aliquot of culture was passaged 5 times during a 2-week period prior to use for experimental inoculation. The final passage was established in antibiotic-free modified Diamond media, and concentration of trichomonads was quantified by use of a hemacytometer. Contaminating bacterial or viral pathogens were not detected during aerobic bacterial culture or electron microscopic examination of a $10-\mu \mathrm{l}$ aliquot of the final inoculum. The cloned 18S rRNA gene of the isolate used for experimental inoculation (NCSU Tfs-1) had 99.9\% sequence identity with $T$ foetus. ${ }^{14,15}$
Inoculation-Each cat was fed 1 tablespoon $(15 \mathrm{ml})$ of a canned commercially available product formulated for cats $s^{c}$ and then sedated, using ketamine hydrochloride (10 to 20 $\mathrm{mg} / \mathrm{cat}$ ) and diazepam ( 0.5 to $1 \mathrm{mg} / \mathrm{cat})$ administered IV. Each cat subsequently received $10 \mathrm{ml}$ of antibiotic-free modified Diamond media containing approximately $2 \times 10^{6}$ trichomonads via orogastric intubation (day 0). Vomiting or other untoward complications associated with administration of the inoculum were not detected.

Data collection-Fecal consistency was recorded daily on the basis of a 4-point scale ( 1 = formed [normal consistency and hard, with distinct or impacted fecal balls]; 2 = semiformed [voluminous, smooth-surfaced, and soft; discrete fecal balls not evident]; 3 = cow pat [not formed, consisting of loose puddles and piles of wet feces]; and 4 = liquid [watery and loose]). Stools were further characterized on the basis of evidence of fresh blood (score, 0.5) and mucus (score, 0.5). Also recorded daily were the total number of bowel movements, number of episodes of vomiting, and a subjective assessment of each cat's appetite and mentation (normal vs abnormal). Body weight and rectal temperature were recorded a minimum of once weekly.

Fecal examinations for Tritrichomonas and Cryptosporidium organisms were performed 1 to 3 times weekly throughout the 29-month study. Two fecal collection methods for recovery of trichomonads were evaluated. Feces were collected per rectum from the proximal portion of the colon, using a plastic loop, or retrieved from the litter pan between 9:00 and 10:00 AM and were immediately processed for examination. Feces from each cat were examined in saline $(0.9 \%$ $\mathrm{NaCl}$ ) solution at $400 \mathrm{X}$ magnification for evidence of trichomonads. If trichomonads were not seen during direct microscopy, feces were cultured in modified Diamond medium. Cultures were incubated at $37 \mathrm{C}$ and monitored daily for evidence of trichomonads by examination of a 10- $\mu$ l aliquot at $400 \mathrm{X}$ magnification. Culture tubes were discarded when growth was not evident after 5 days. A thin smear of feces from each cat was stained with Auramine- 0 and examined under a fluorescence microscope for evidence of Cryptosporidium oocysts. Oocysts were semiquantified on a scale $^{19}$ of 0 to 4 by a single examiner (MGL) who was not aware of the identity and treatment status of any cat.

A CBC, serum biochemical analyses, and urinalysis were performed on samples obtained from all cats on days $-14,50$, and 203. On days $-7,30$, and 121 , intestinal biopsy specimens were obtained from all cats. Prior to intestinal biopsy, food was withheld from each cat for 12 hours, and each cat was given 2 warm water enemas. Subsequently, cats were sedated (ketamine [ 10 to $20 \mathrm{mg} / \mathrm{cat}$ ] and diazepam [0.5 to 1 $\mathrm{mg} / \mathrm{cat}], \mathrm{IV})$. Colonic mucosal suction biopsy specimens were obtained from the proximal, middle, and distal portions of the colon. Mucosal samples were fixed in neutral-buffered $10 \%$ formalin, routinely processed, paraffin embedded, sectioned at a thickness of $5 \mu \mathrm{m}$, and stained with H\&E for examination by use of light microscopy. Mucosal inflammation in each region of the colon was semiquantified by a single examiner (JML) who was unaware of the location of the biopsy specimen, time of collection of the biopsy specimen, and identity of each cat. Scoring of inflammation was as follows: 0 , normal; 1 , very mild; 2 , mild; 3 , moderate; 4 , moderately severe; and 5 , severe.

Evaluation of treatments-Two treatments were evaluated. All group-1 cats (Cryptosporidium coinfected) received NTZ ( $75 \mathrm{mg} / \mathrm{kg}$ of body weight, PO, q $24 \mathrm{~h}$ beginning on day 49). Tablets of NTZ ${ }^{\mathrm{g}}$ were ground to a fine powder and loaded into No. 3 gelatin capsulses to achieve an exact dose based on each cat's body weight. Vomiting, which coincided with drug administration, necessitated discontinuation of 
treatment after 5 days. Treatment was reinstituted 1 week later at a lower dosage $(25 \mathrm{mg} / \mathrm{kg}, \mathrm{PO}, \mathrm{q} 12 \mathrm{~h})$ administered in combination with the antiemetic chlorpromazine ( 0.5 to $1.5 \mathrm{mg}, \mathrm{SC}, \mathrm{q} 24 \mathrm{~h}$ ). Both drugs were administered for 28 days. While receiving NTZ, group-1 cats had additional CBC, serum biochemical analyses, and urinalyses performed on days 53, 77, and 92 to screen for adverse effects attributable to drug administration. Group- 1 and -2 cats received $10 \mathrm{mg}$ of prednisolone ( 2.8 to $3.8 \mathrm{mg} / \mathrm{kg}, \mathrm{PO}, \mathrm{q} 24 \mathrm{~h}$ for 26 days) beginning on day 133 .

Identification $T$ foetus in feces from experimentally infected cats-Briefly, DNA was extracted, and the 18S rRNA gene was amplified and cloned, using commercially available kits. $^{\text {h-j }}$ Sequencing was performed ${ }^{\mathrm{k}}$ and analyzed, using a computerized program. ${ }^{1}$ Results were compared with known sequences available through a database. ${ }^{\mathrm{m}}$

Necropsy-On day 203, cats were euthanatized by injection of pentobarbital sodium $(17.7 \mathrm{mg} / \mathrm{kg}$, IV). Samples were immediately obtained for culture of trichomonads from specific sites beginning in the oral cavity and extending to the distal portion of the colon of each cat. Samples included swab specimens of the gingival, sublingual, and pharyngeal mucosa and samples $(0.1 \mathrm{ml})$ of luminal contents from the stomach, duodenum, jejunum (at a point $50 \mathrm{~cm}$ proximal to the rectum), ileum, cecum, proximal portion of the colon, distal portion of the colon, gall bladder, and urinary bladder. Full-thickness tissue samples were obtained from the cecum as well as the proximal, middle, and distal portions of the colon. Tissue samples were fixed in neutral-buffered $10 \%$ formalin, routinely processed, paraffin embedded, sectioned at a thickness of $5 \mu \mathrm{m}$, and stained with $\mathrm{H} \& \mathrm{E}$ for examination, using light microscopy. Mucosal inflammation was scored as described previously for biopsy specimens. The pathologist was unaware of the location of the biopsy specimen and identity of each cat; however, time at which the specimen was obtained (ie, during necropsy) was apparent by the fullthickness nature of these samples.

Immunohistochemical analysis for $T$ foetus-Mucosal biopsy specimens from cats experimentally inoculated with $T$ foetus were fixed in neutral-buffered $10 \%$ formalin for 24 hours, embedded in paraffin, sectioned at a thickness of 5 $\mu \mathrm{m}$, and mounted on poly-L-lysine-coated slides. Tissues were deparaffinized by immersion in xylene, rehydrated in a graded series of ethanol solutions, and hydrated with buffer (PBS solution, $\mathrm{pH}$ 7.4). Tissues were treated with $3 \% \mathrm{H}_{2} \mathrm{O}_{2}$ in methanol for 10 minutes at $4 \mathrm{C}$ to quench endogenous peroxidase and were blocked for 30 minutes at $22 \mathrm{C}$ with nonimmune goat serum. A commercial kit ${ }^{\mathrm{n}}$ was used for blocking endogenous avidin and biotin activity, and tissues were incubated with a monoclonal antibody ${ }^{\circ}$ (TF1.15) that recognizes a surface antigen of $T$ foetus. ${ }^{20}$ The antibody was diluted 1:500 with diluent $\mathrm{t}^{\mathrm{p}}$ before incubation (60 minutes at 37 C). Sections were immunostained, using a commercially available broad spectrum streptavidin-biotin-peroxidase system with 3-amino-9-ethylcarbazole (AEC) as the chromogen. ${ }^{q}$ Sections were counterstained with methyl green for 30 seconds and rinsed in tap water. Coverslips were mounted with an aqueous mounting media. Negative-control sections were not treated with primary antibody.

Statistical analysis-All statistical analyses were performed, using a commercial software package. ${ }^{r}$ The MannWhitney rank sum test was used to detect differences between group-1 and -2 cats for scores for fecal consistency 0 to 7 weeks after inoculation, frequency of defecation, and number of positive results for direct microscopic examinations of Tritrichomonas spp 0 to 203 days after inoculation; that test also was used to determine differences between methods of feces collection (loop vs voided) for detection of Tritrichomonas spp by direct microscopic examination or culture 0 to 203 days after inoculation and fecal oocyst scores of group-1 cats prior to and following inoculation with $T$ foetus until treatment with NTZ. Repeated-measures ANOVA on ranks was used to compare scores for mucosal inflammation among regions of the colon at a single time period and within a region of the colon among time periods. For all analyses, values of $P<0.05$ were considered significant.

\section{Results}

Experimentally induced infection with $T$ foetusBeginning 2 to 7 days after orogastric inoculation, direct examination or protozoal culture of feces from each cat revealed infection with Tritrichomonas spp. Infection persisted in all cats throughout the entire 203 days of the study. It was confirmed, using 185 rRNA gene sequence analysis, that axenic cultures of Tritrichomonas spp reisolated from experimentally

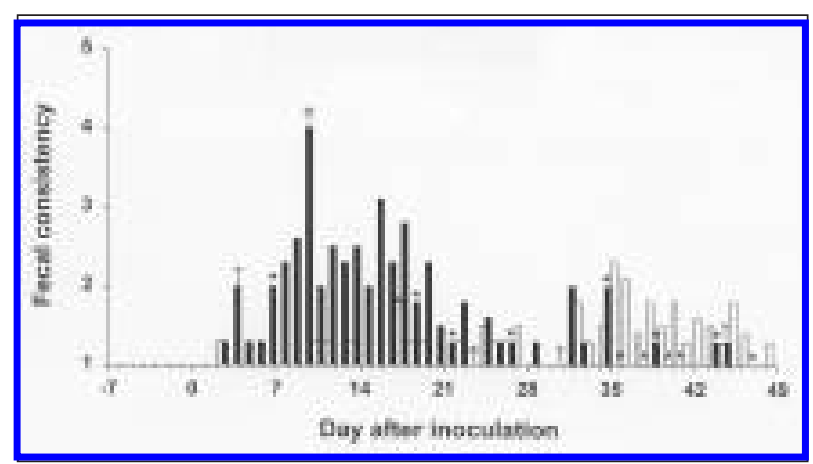

Figure 1-Mean scores of fecal consistency for cats experimentally inoculated with Tritrichomonas foetus. Four cats naturally coinfected with Cryptosporidium spp (black bar) and 4 specific-pathogen-free cats (white bar) were orogastrically infected (day 0) with $2 \times 10^{6} T$ foetus organisms (NCSU Tfs-1). Fecal consistency was scored as follows: $1=$ formed, 2 = semiformed, $3=$ cow pat, and $4=$ liquid. It was further characterized by evidence of fresh blood (score, 0.5) and mucus (score, 0.5).

* Mean score for only 3 cats. †Mean score for only 2 cats. ¥Score for only 1 cat.

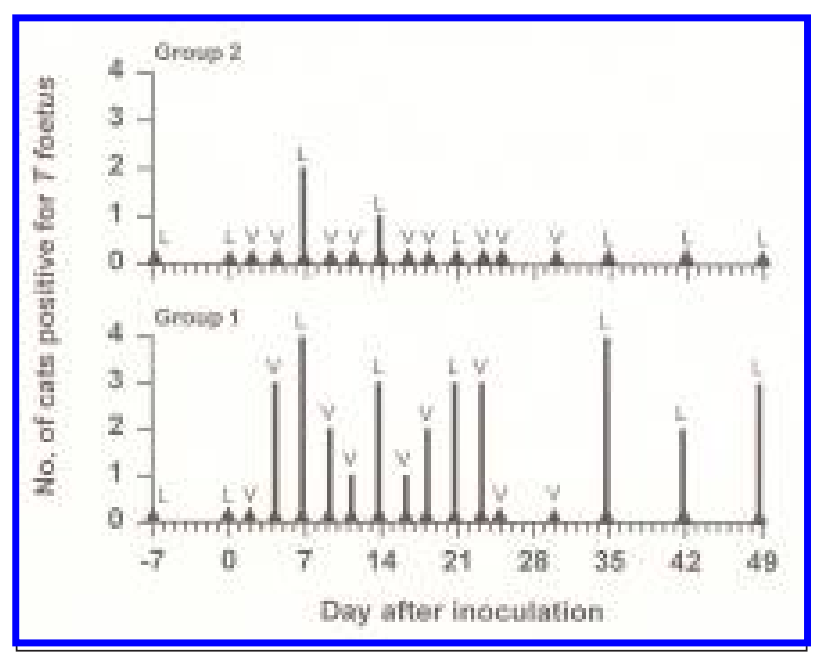

Figure 2-Number of cats with positive results for $T$ foetus by direct microscopic examination of feces. Fecal samples were obtained after natural voiding (V) or collected from the rectum by use of a plastic loop (L). All cats were experimentally inoculated on day 0 . Group 1 = Cats coinfected with Cryptosporidium spp $(n=4)$. Group 2 = Specific-pathogen-free cats $(n=4)$. 
infected cats contained $T$ foetus that had $100 \%$ sequence identity with that of the inoculated isolate. Infection with $T$ foetus induced diarrhea in all cats in both groups. Onset of diarrhea was significantly $(P=$ 0.02 ) earlier and was of greater severity in group-1 cats, compared with group-2 cats (Fig 1). Diarrhea varied in consistency from semiformed to cow pat, and fresh blood was seen infrequently in feces from 4 cats (2 from each group). Within group 1, onset of diarrhea was not associated with significant changes in the number of fecal Cryptosporidium oocysts. For both groups, fecal consistency returned to normal (fecal score of 1) approximately 7 weeks after inoculation; however, semiformed feces were periodically observed thereafter in all cats. In addition, all cats developed diarrhea for a period of 5 to 8 days after each colonic biopsy. We did not detect significant changes in defecation frequency, rectal temperature, appetite, body weight or attitude, or episodes of vomiting associated with infection with $T$ foetus.

Fecal analysis of $T$ foetus-infected cats-Fecal samples collected by use of a loop had positive results significantly $(P=0.025)$ more frequently during direct microscopy, compared with results for voided samples. However, throughout the study, T foetus was cultured with equal efficacy from feces collected by either technique. Fecal samples from diarrheic cats were better than formed feces when using direct microscopic examination was used for detection of infection with $T$ foetus. During direct microscopic examination, feces from group- 2 cats were virtually devoid of $T$ foetus after resolution of the initial 7-week period of diarrhea, and organisms were not seen during episodes of biopsyassociated diarrhea. However, group-1 cats continued to shed detectable numbers of $T$ foetus despite resolution of diarrhea $(P<0.001 ;$ Fig 2$)$ and after periods of colonic biopsy.

Effect of NTZ on infections attributable to $T$ foetus and Cryptosporidium spp-Treatment of group-1 cats with NTZ (25 mg/kg, PO, q $12 \mathrm{~h})$ was associated with dark brown-to-black foul-smelling diarrhea that began to abate after day 21 of the 28-day course of treatment. Vomiting, which was observed in all treated cats, was evident several hours after drug administration and was decreased with chlorpromazine administration. Hematologic or biochemical abnormalities were not identified during or following treatment. After day 14 of treatment with NTZ, all group-1 cats had negative results for $T$ foetus when tested by use of direct fecal examination and microbial culture. However, during a 10-day period after the final dose of NTZ, all group-1 cats had diarrhea (semiformed to cow pat, with and without fresh blood) in which the feces contained microscopically detectable $T$ foetus (Fig 3). Group-2 cats, which did not receive NTZ, remained healthy with predominantly formed but culture-positive feces throughout this period. Treatment with NTZ was associated with an immediate and sustained termination of shedding of Cryptosporidium oocysts by all group-1 cats (Fig 4).

Effect of prednisolone on infection attributable to $T$ foetus and Cryptosporidium spp-Treatment of group1 and -2 cats with prednisolone (10 mg, PO, q $24 \mathrm{~h}$ for 26 days) did not have an effect on fecal consistency, defecation frequency, or results of direct fecal examination or microbial culture for $T$ foetus. Both groups of cats remained clinically normal with predominantly formed feces. Results of direct fecal examinations were negative, but $T$ foetus was cultured routinely throughout this period. In contrast to its lack of effects on infection attributable to $T$ foetus, prednisolone treatment was associated with recrudescent shedding of Cryptosporidium oocysts in 2 cats in group 1 (Fig 4).

Histologic examination and gastrointestinal distribution of $T$ foetus-Prior to inoculation with $T$ foetus and throughout the study, mean mucosal inflammation scores ranged from extremely mild to mild and consisted of small numbers of lymphocytes, plasma cells, and a

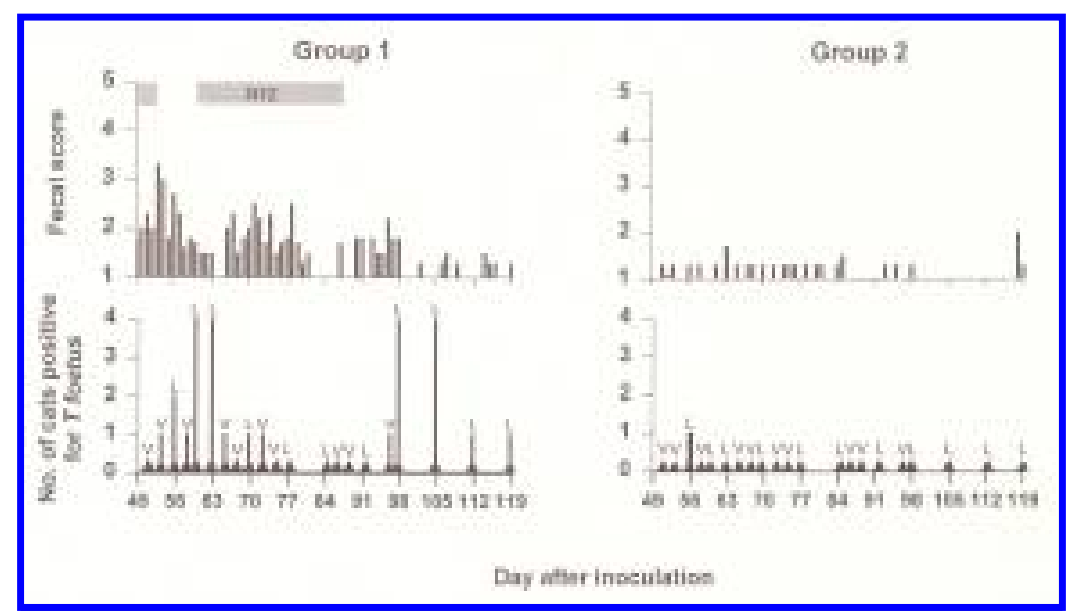

Figure 3-Mean fecal consistency scores and results of direct microscopic examination of feces from 2 groups of cats experimentally inoculated with $T$ foetus. Group-1 cats were naturally coinfected with Cryptosporidium spp and were treated with nitazoxanide (NTZ) during specified periods (gray bars). Group-2 cats (treatmentcontrol cats) were not coinfected with Cryptosporidium spp and did not receive NTZ. See Figures 1 and 2 for key. 
few neutrophils. We did not detect significant differences in histologic scores among various segments of the colon, among various time frames after inoculation, or between group-1 and -2 cats. Immunohistochemical analysis for T foetus antigen in neutral-buffered $10 \%$ formalin-fixed tissue sections of cecal and colonic mucosa from experimentally inoculated cats was used to identify trichomonads and antigen within superficial mucus and detritus and in association with surface epithelia. Tritrichomonas foetus antigen also was detected within surface enterocytes in those areas in which there were numerous luminal organisms (Fig 5). Organisms were not seen within the crypts or lamina propria. Positive immunolabeling was not evident in biopsy specimens collected prior to experimental inoculation or in sections in which use of primary antibody was omitted (negative-control specimens).

During postmortem examination, $T$ foetus was cultured from the contents of ileum (3 cats), cecum (5), proximal portion of the colon (5), and rectum (8). There were not apparent differences in anatomic location of infection between group-1 and -2 cats. Tritrichomonas foetus was not cultured from the oral cavity, stomach, duodenum, jejunum, bile, or urine of any cat.

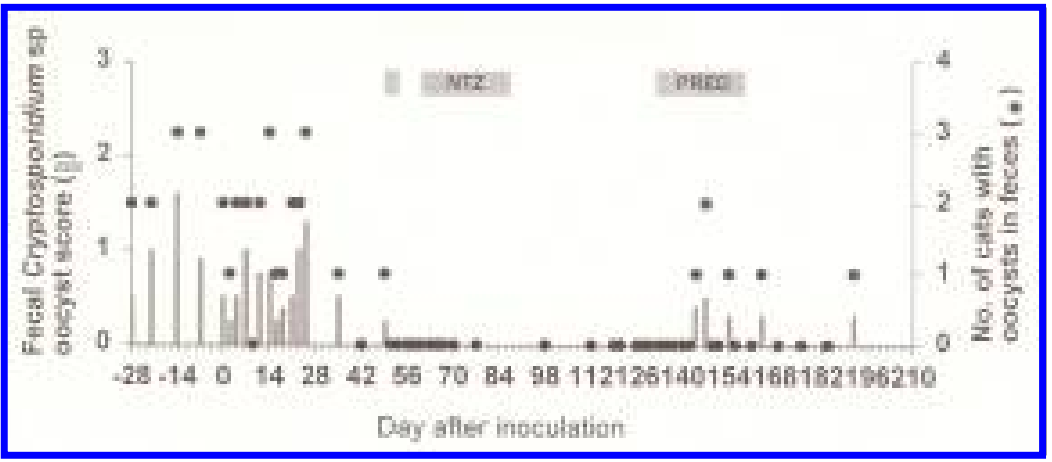

Figure 4-Depiction of the natural course of cryptosporidiosis in 4 group-1 cats on the basis of excretion of Cryptosporidium oocysts in feces. Number of oocysts was semi-quantified on a scale from 0 to 4 , and the number of cats shedding oocysts was recorded. Cats were treated with NTZ and prednisolone (Pred) during specified periods (gray bars).

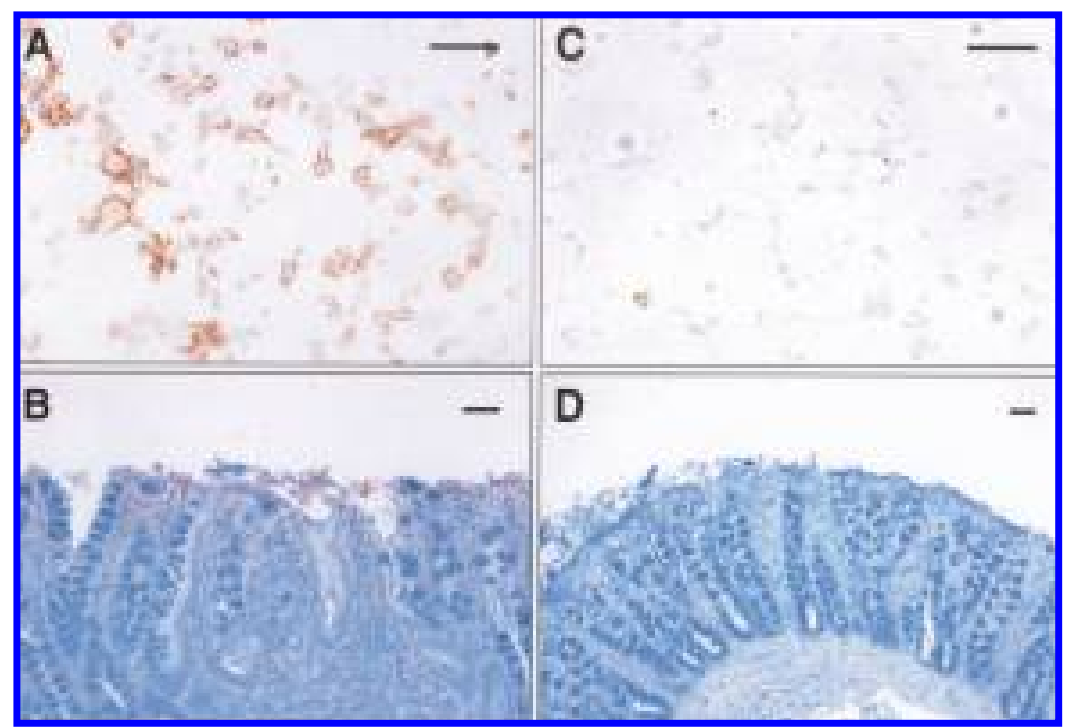

Figure 5-Photomicrograph of immunohistochemical analysis for $T$ foetus antigen in colonic mucosal biopsy specimens obtained from cats, using monoclonal antibody TF1.15 (dilution of 1:500, incubated 60 minutes at $37 \mathrm{C}$ ). Panels $A$ and $C$ are cytocentrifuged preparations of $T$ foetus (NCSU Tfs-1) cultured from a cat with largebowel-type diarrhea. Trichomonads were fixed in formalin and centrifuged ( $300 \times \mathrm{g}$ for 5 minutes) onto poly-L-lysine-coated slides prior to immunohistochemical staining. Slides were treated identically, except for omission of primary antibody for panel $\mathrm{C}$ (negative-control specimen). Surface immunolabeling of trichomonads is evident in panel A, but notice that background staining is not evident in panel C. Panels B and $D$ are similar tissue sections obtained from a cat experimentally inoculated with $T$ foetus. Sections were treated identically, except for omission of primary antibody for panel D (negative-control specimen). In panel B, immunolabeled trichomonads (redstained organisms) are located on surface enterocytes and within superficial mucus and detritus of the cecal mucosa. Antigen also is evident within the superficial epithelia and lamina propria. Notice that background staining is not evident in panel D. Immunostained by streptavidin-biotin-peroxidase with 3-amino-9-ethylcarbazole as the chromogen and methyl green counterstain. Bar $=100 \mu \mathrm{m}$. 


\section{Discussion}

Recent reports ${ }^{11-13}$ have implicated an association between intestinal trichomonosis and large-bowel diarrhea in young cats. Efforts to cultivate trichomonads from the feces of healthy indoor and feral cats from the same geographic region as that of infected cats have been unsuccessful, ${ }^{11}$ suggesting that the trichomonads are not a component of the normal intestinal fauna. In the study reported here, T foetus (NCSU Tfs-1) was isolated from the feces of a naturally infected kitten with a history of chronic large-bowel diarrhea. Orogastric inoculation of SPF cats with axenic cultures of this $T$ foetus isolate resulted in infection of the ileum and colon for at least 203 days and diarrhea for at least 7 weeks. Tritrichomonas foetus with $100 \%$ sequence identity to the infecting isolate was recultured from 1 of the experimentally inoculated cats. These observations fulfill Koch's postulates and suggest that $T$ foetus is a previously unrecognized intestinal pathogen of cats.

Several prior and current observations suggest that the colonic microflora play a role in the pathogenesis of $T$ foetus-related diarrhea. Cats naturally infected with $T$ foetus typically have a waxing and waning course of diarrhea. ${ }^{11-13}$ In the absence of diagnostic or therapeutic interventions, naturally infected cats may undergo prolonged periods of clinical remission. Fecal consistency may improve during treatment with various antimicrobials; however, diarrhea recurs and often is worse when treatment is discontinued. ${ }^{11}$ In the study reported here, diarrhea (fecal scores $>1$ ) resolved 7 weeks after inoculation; however, acute self-limiting diarrhea recurred after administration of an enema and performance of colonic biopsy and after discontinuing treatment with the antimicrobial drug NTZ. These findings suggest that fluctuations in the colonic microflora or other undetermined factors may be necessary to produce the clinical manifestations of $T$ foetus infection. A close relationship between $T$ foetus and the colonic microflora is supported by the failure of early attempts to cultivate trichomonads in the absence of bacteria. ${ }^{21}$ It is now understood that trichomonads are obligate parasites that depend on endogenous bacterial flora and host secretions for acquisition of essential nutrients. For successful culture, the isolation media must contain all of these components. ${ }^{21}$ On the basis of the study reported here, there does not appear to be a simple relationship between $T$ foetus and diarrhea, because the organism can persist in cats without diarrhea, and diarrhea can become exacerbated without an apparent increase in the number of organisms in the intestinal tract.

Following inoculation with $T$ foetus, cats with coexisting cryptosporidiosis (group 1) developed diarrhea earlier and with greater severity than SPF cats (group 2). It is unlikely that this difference reflected a natural worsening of cryptosporidial infection in group-1 cats. Group-1 cats consistently shed small numbers of oocysts and did not have clinical signs of cryptosporidiosis for 4 weeks prior to inoculation with $T$ foetus. Furthermore, there was not a detectable change in the number of oocysts shed after inoculation with $T$ foetus; cryptosporidia were not seen in any colonic mucosal biopsy specimens, and large-bowel diarrhea would not be expected, because Cryptosporidium spp have a trophism for the small intestine. Whereas prednisolone caused recrudescence of shedding of Cryptosporidium oocysts in 2 cats, there was not a detectable change in consistency of their feces, which appeared normal. After resolution of the initial 7 -week period of diarrhea, $T$ foetus was still commonly observed in fecal smears of samples obtained from group-1 cats but not in fecal smears obtained from group- 2 cats. Although the reason for this difference was unresolved by the design of this study, analysis of these results suggested that coexisting enteric infection with Cryptosporidium spp was associated with proliferation of $T$ foetus. Because fecal consistency was normal in both groups, it is unlikely that the coexisting infection merely promoted passage of $T$ foetus into feces.

Tritrichomonas foetus appears to establish a chronic experimentally induced infection that is restricted to the ileum, cecum, and colon. Although clinical signs may periodically remiss, we have not observed spontaneous resolution of infection in any experimentally or naturally infected cats that have been evaluated by means of fecal culture for Tritrichomonas organisms. In the study reported here, very mild inflammation was observed in colonic mucosal biopsy specimens obtained from cats prior to experimental inoculation. These findings may have been normal for this group of cats, because our evaluations prior to onset of the study were extensive and did not reveal other diagnostic or clinical evidence of underlying colonic disease. Importantly, substantial colonic inflammation was not observed during the 203-day experimental period after inoculation. Only a few naturally infected cats have had colonic biopsy specimens obtained for further evaluation of large-bowel diarrhea, and all have revealed varying degrees of lymphocytic-plasmacytic inflammation. ${ }^{11}$ Using a $T$ foetus-specific monoclonal antibody, ${ }^{20}$ trichomonads were found in close association with the surface epithelia and mucus of the cecal and colonic mucosa. Evidence of mucosal invasion by intact $T$ foetus was not observed. The positive immunolabeling of enterocytes seen in proximity to overlying $T$ foetus in these experimentally infected cats also has been observed in preputial epithelium of naturally infected bulls. ${ }^{22}$ In bulls, this observation was attributed to antigen uptake and translocation by the surface epithelium. ${ }^{22}$ Whether more chronic trichomonosis of the colon of cats leads to subsequent development of inflammatory bowel disease cannot be answered by the study reported here.

For direct microscopic detection of $T$ foetus, fresh feces collected by use of a plastic loop yielded more positive results than did voided samples, probably because the organism survives better in fresh feces or because the samples obtained with the loop were collected from a location that was more proximal. When results of direct fecal examination are negative, particularly if feces are formed and voided, fecal culture for Tritrichomonas spp should be used to exclude the possibility of infection with $T$ foetus.

Nitazoxanide is a nitrothiazole benzamide compound (20[acetolyloxy]-N-[5-nitro-2-thiazolyl] benza- 
mide) and, in combination with its desacetyl derivative, tizoxanide, has the broadest spectrum of antiparasitic activity (flagellate, ciliate, and coccidial protozoa, nematodes, cestodes, and trematodes) achieved by a single drug. ${ }^{18}$ The mechanism of action of NTZ is unknown; however, reduction of the nitro group followed by binding to DNA of the target pathogen has been hypothesized. Pertinent to our study are reports of in vitro activity of NTZ against $T$ vaginalis,${ }^{23}$ variable efficacy against Cryptosporidium spp, ${ }^{23-27}$ and a single report ${ }^{28}$ on the use of NTZ for treatment of cats with intestinal parasites. Among numerous antimicrobial drugs tested, NTZ was identified as the sole drug capable of inhibiting in vitro growth of our $T$ foetus isolate (NCSU Tfs-1). ${ }^{\mathrm{a}}$

All cats vomited and had diarrhea during treatment with NTZ. Vomiting was alleviated by chlorpromazine, a central-acting antiemetic. During treatment with NTZ, feces were dark brown-to-black, foulsmelling, and loose but contained progressively fewer $T$ foetus organisms. Diarrhea was suspected to have resulted from the broad-spectrum antimicrobial effects of NTZ on normal intestinal bacterial flora. ${ }^{18}$ In the sole prior report ${ }^{28}$ cats developed mild diarrhea after a single dose of NTZ (100 mg/kg, PO). Drug-induced diarrhea also has been reported in healthy gnotobiotic baby pigs treated with NTZ. ${ }^{24}$ Of potential importance, we have determined that $T$ foetus reisolated from cats treated with NTZ are no longer susceptible to the drug in vitro. ${ }^{a}$ Therefore, in the study reported here, failure of NTZ to fully eradicate $T$ foetus was suggestive of acquired drug resistance rather than inadequate administration of treatment.

In contrast to temporary suppression of $T$ foetus infection that results from prolonged administration of treatment, the first dose of NTZ given $(75 \mathrm{mg} / \mathrm{kg}, \mathrm{PO})$ was followed by an immediate and sustained period in which Cryptosporidium oocysts were not detected in the feces. Because our untreated infection control group (group 2) did not have concurrent infection with Cryptosporidium spp, the possibility of coincidental remission of oocyst shedding cannot be ruled out. Nitazoxanide did not appear to eradicate Cryptosporidium infection, because administration of prednisolone resulted in excretion of oocysts in 2 of 4 cats. Because of strict sanitation and housing conditions, reinfection of these 2 cats with Cryptosporidium spp was considered unlikely.

It is not known whether immunosuppression plays a role in the predisposition of cats to chronic infection with $T$ foetus. Other than their young age and often stressful multiple-cat environment, concurrent immunosuppressive diseases (eg, FeLV or FIV) have not been a feature of naturally occurring $T$ foetus infection. ${ }^{11-13}$ In the study reported here, immunosuppressive treatment with prednisolon $e^{29}$ did not have an effect on fecal consistency or the frequency of positive results of direct fecal examinations for Tritrichomonas organisms. However, quantitative cultures of $T$ foetus were not performed. Results of this study do not provide insight into the potential outcome of corticosteroid administration in cats with concurrent inflammatory bowel disease and infection with $T$ foetus, because these cats did not have diarrhea or substantial colonic inflammation at the time of prednisolone administration.

Results of the study reported here revealed that after experimental inoculation, $T$ foetus persistently colonize the feline ileum, cecum, and colon, where they reside within superficial mucus and cellular debris and in contact with the epithelium. Infection attributable to $T$ foetus can be diagnosed by means of direct microscopic examination of fresh diarrheal feces or by culture of feces in modified Diamond media. Experimental inoculation resulted in a noninflammatory large-bowel diarrhea that could be exacerbated by coexisting infection with Cryptosporidium spp but not by administration of an immunosuppressive dosage regimen of prednisolone. An effective antimicrobial treatment for cats infected with $T$ foetus has yet to be identified.

Tritrichomonas foetus is the causative organism of venereal trichomonosis in cattle, and $T$ suis is the trichomonad found in the nasal mucosa and intestinal tract of swine. ${ }^{16,17}$ These organisms are considered to be identical on the basis of morphology, ultrastructural analysis, isozymic homogeneity, and analysis of the 5.8S rRNA gene sequence. ${ }^{17,30}$ Furthermore, experimental infection of various hosts with $T$ foetus or $T$ suis has revealed little host specificity. ${ }^{31,32}$ The natural history of $T$ foetus infection in cats and any relationship to trichomonosis in cattle or pigs are unknown. In light of the relative lack of host specificity of this organism and the intimate association between infected cats and their human companions, the potential for zoonotic transmission should be considered.

${ }^{\mathrm{a}}$ Levy MG, Gookin JL, Poore MF, et al. Intestinal trichomonosis in cats: pathology, diagnosis and susceptibility to antiprotozoal drugs (abstr), in Proceedings. Joint Meet Am Soc Parasitol Soc Protozool 2000;108

'Liberty Research Inc, Waverly, NY.

'Hills' Science Diet Feline Growth, Hills' Nutrition, Topeka, Kan.

${ }^{d}$ ProSpecT Giardia rapid assay, Alexon-Trend Inc, Ramsey, Minn.

'Modified Diamond medium, Remel, Lenexa, Kan.

${ }^{r}$ Model $4.7 \mathrm{~mm}$ X $120 \mathrm{~cm}$, Quinton Instrument Co, Seattle, Wash.

${ }^{8}$ Courtesy of Blue Ridge Pharmaceuticals, Greensboro, NC.

hDNeasy tissue kit, Qiagen, Valencia, Calif.

'TOPO TA cloning kit, Invitrogen, Carlsbad, Calif.

'Qiaprep Spin Miniprep kit, Qiagen, Valencia, Calif.

kAutomatic DNA sequencing facility, University of North Carolina, Chapel Hill, NC.

'Wisconsin package, version 10.0, Genetics Computer Group, Madison, Wis.

${ }^{\mathrm{m} N C B I}$ GENBANK (database online). Available at http://www. ncbi.nlm.nih.gov/BLAST/. Accessed June 2000.

"Avidin/Biotin blocking kit, Zymed Laboratories Inc, San Francisco, Calif.

${ }^{\circ}$ MabTF1.15, courtesy of Corbeil LB, Department of Pathology, School of Medicine, University of California, San Diego, Calif.

${ }^{\mathrm{P} A n t i b o d y}$ diluent reagent solution, Zymed Laboratories Inc, San Francisco, Calif.

${ }^{\mathrm{q}}$ Histostain-SP broad spectrum, Zymed Laboratories Inc, San Francisco, Calif.

'SigmaStat statistical software, Jandel Scientific, San Rafael, Calif.

\section{References}

1. Da Cunha AM, Muniz J. Trabalhos do Instituto Oswaldo Cruz. Sobre um flagellado parasito do gato. Brazil-Medico 1922;36:285-286.

2. Brumpt E. Recherches morphologiques et experimentales sur le Trichomonas felis Da Cunha et Muniz, 1922, parasite du chat et du chien. Ann Parasitol 1925;3:239-251.

3. Simic TSH. Etude biologique et experimentale du 
Trichomonas intestinalis, infectant spontanement l'homme, le chat et le chien. Ann Parasitol 1932;10:209-224.

4. Kessel JF. Trichomoniasis in kittens. Trans $R$ Soc Trop Med Hyg 1928;22:61-80.

5. Hegner R, Eskridge L. Absence of pathogenicity in cats infected with Trichomonas felis from cats and Trichomonas hominis from man. Am J Hyg 1935;22:322-325.

6. Burrows CF, Batt RM, Sherding RG. Diseases of the small intestine. In: Ettinger SJ, Feldman EC, eds. Textbook of veterinary internal medicine. 4th ed. Philadelphia: WB Saunders Co, 1995;1202.

7. Barr SC. Enteric protozoal infections. In: Greene CE, ed. Infectious diseases of the dog and cat. 2nd ed. Philadelphia: WB Saunders Co, 1998;487.

8. Dimski DS. Helminth and noncoccidial protozoan parasites of the gastrointestinal tract. In: Sherding RG, ed. The cat: diseases and clinical management. 2nd ed. New York: Churchill Livingstone Inc, 1989;459-477.

9. Guilford WG, Strombeck DR. Gastrointestinal tract infections, parasites, and toxicoses. In: Strombeck's small animal gastroenterology. 3rd ed. Philadelphia: WB Saunders Co, 1996;427.

10. Jordan HE. Trichomonas spp. in feline: a case report. Vet Med 1956;51:23-24.

11. Gookin JL, Breitschwerdt EB, Levy MG, et al. Diarrhea associated with trichomonosis in cats. IAm Vet Med Assoc 1999;215:1450-1455.

12. Romatowski J. An uncommon protozoan parasite (Pentatrichomonas hominis) associated with colitis in three cats. Feline Pract 1996;24:10-14.

13. Romatowski J. Pentatrichomonas hominis infection in four kittens. IAm Vet Med Assoc 2000;216:1270-1272.

14. Levy MG, Gookin JL, Poore MF, et al. Information on parasitic gastrointestinal infections in cats (lett). L Am Vet Med Assoc 2001;218:194-195.

15. Levy MG, Gookin JL, Poore MF, et al Tritrichomonas foetus and not Pentatrichomonas hominis is the causative agent of the recently described feline intestinal trichomonosis. J Vet Intern Med 2001;15:315.

16. Pakandl M. The prevalence of intestinal protozoa in wild and domestic pigs. Vet Med (Praha) 1994;39:377-380.

17. Felleisen RSJ. Comparative sequence analysis of $5.8 \mathrm{~S}$ rRNA genes and internal transcribed spacer (ITS) regions of trichomonadid protozoa. Parasitology 1997;115:111-119.

18. Dubreuil L, Houcke I, Mouton Y, et al. In vitro evaluation of activities of nitazoxanide and tizoxanide against anaerobes and aerobic organisms. Antimicrob Agents Chemother 1996;40:2266-2270.

19. Levy MG, Ley DH, Barnes HJ, et al. Experimental cryp- tosporidiosis and infectious bursal disease virus infection of specificpathogen-free chickens. Avian Dis 1988;32:803-811.

20. Hodgson JL, Jones DW, Widders PR, et al. Characterization of Tritrichomonas foetus antigens by use of monoclonal antibodies. Infect Immunol 1990;58:3078-3083.

21. Diamond LS. The establishment of various trichomonads of animals and man in axenic cultures. I Parasitol 1957;43:488-490.

22. Rhyan JC, Wilson KL, Wagner B, et al. Demonstration of Tritrichomonas foetus in the external genitalia and of specific antibodies in preputial secretions of naturally infected bulls. Vet Pathol 1999;36:406-411.

23. Broekhuysen J, Stockis A, Lins RL, et al. Nitazoxanide: pharmacokinetics and metabolism in man. Int I Clin Pharmacol Ther 2000;38:387-394.

24. Theodos CM, Griffiths JK, D'Onfro J, et al. Efficacy of nitaz oxanide against Cryptosporidium parvum in cell culture and in animal models. Antimicrob Agents Chemother 1998;42:1959-1965.

25. Giacometti A, Cirioni O, Barchiesi F, et al. Activity of nitaz oxanide alone and in combination with azithromycin and riabutin against Cryptosporidium parvum in cell culture. I Antimicrob Chemother 2000;45:453-456.

26. Blagburn BL, Drain KL, Land TM, et al. Comparative efficacy evaluation of dicationic carbazole compounds, nitazoxanide, and paromomycin against Cryptosporidium parvum infections in a neonatal mouse model. Antimicrob Agents Chemother 1998;42: 2877-2882.

27. Giacometti A, Burzacchini F, Cirioni O, et al. Efficacy of treatment with paromomycin, azithromycin, and nitazoxanide in a patient with disseminated cryptosporidiosis. Eur I Clin Microbiol Infect Dis 1999;18:885-889.

28. Euzeby J, Tep SP, Rossignol JF. Experimentation des proprietes anthelminthiques de la nitazoxanide chez le chien, le chat et les ovins. Res Med Vet 1980;131:687-696.

29. Plumb DC. Prednisolone. In: Veterinary drug handbook. 3rd ed. Ames, Iowa: Iowa State University Press, 1999;533.

30. Mattos A, Sole-Cava AM, DeCarli G, et al. Fine structure and isozymic characterization of trichomonadid protozoa. Parasitol Res 1997;83:290-295.

31. Kerr WR. Experiments in cattle with Trichomonas suis. Vet Rec 1958;70:613-615.

32. Fitzgerald PR, Johnson AE, Hammond DM, et al. Experimental infection of young pigs following intranasal inoculation with nasal, gastric, or cecal trichomonads from swine or with Trichomonas foetus. IParasitol 1958;44:597-602. 